\title{
The concept of thin film bulk acoustic resonators as selective $\mathrm{CO}_{2}$ gas sensors
}

\author{
Romy Hoffmann ${ }^{1,2}$, Matthias Schreiter ${ }^{1}$, and Johannes Heitmann ${ }^{2}$ \\ ${ }^{1}$ Siemens Corporate Technology, 81739 Munich, Germany \\ ${ }^{2}$ Institute for Applied Physics, TU Bergakademie Freiberg, 09599 Freiberg, Germany \\ Correspondence to: Romy Hoffmann (romy.hoffmann@siemens.com)
}

Received: 29 September 2016 - Revised: 15 December 2016 - Accepted: 9 January 2017 - Published: 13 February 2017

\begin{abstract}
Carbon dioxide $\left(\mathrm{CO}_{2}\right)$ is a gas that well represents air quality in indoor environments as well as being an important greenhouse gas. However, the reliable and affordable sensing of environmental $\mathrm{CO}_{2}$ at room temperature, with techniques other than optical spectroscopy, remains an unsolved problem to this day. One major challenge for solid state sensors is the realisation of adequate selectivity, especially towards changing humidity. The thin film bulk acoustic resonator (FBAR) is a MEMS (Microelectromechanical systems) device that can not only detect gas-induced mass changes but also changes in the acoustic velocity and density of its layers. This multi-sensing provides a suitable platform for selective gas sensing. In this work we present studies done on polyaminosiloxane- and ethyl cellulose-functionalised FBARs regarding $\mathrm{CO}_{2}$ sensitivity, selectivity towards humidity, and stability. We demonstrate how $\mathrm{CO}_{2}$ and humidity signals can be separated and that $\mathrm{CO}_{2}$ can be sensed with a resolution of $50 \mathrm{ppm}$ between 400 and $1000 \mathrm{ppm}$. Using the Mason model, we show how the acoustic velocity and density of an absorption layer can be determined and how changes in those parameters affect the resonance frequency shift. The understanding of these results ultimately presents a tool to theoretically separate any number of gas analytes.
\end{abstract}

\section{Introduction}

The sensing of carbon dioxide $\left(\mathrm{CO}_{2}\right)$ content in air is of interest for many different reasons. For one, it is one of the main greenhouse gases emitted through human activities and since the Kyoto protocol of 1997, efforts have risen to monitor and reduce greenhouse gases in order to scale down climate change and all of its negative consequences. Furthermore, $\mathrm{CO}_{2}$ levels are a good indicator for indoor air quality. Kajtár et al. (2006) found that the concentration of $\mathrm{CO}_{2}$ in closed rooms should be kept lower than $3000 \mathrm{ppm}$ in order to ensure human well-being. The building industry is very interested in knowing the indoor air quality. It gives information about the necessity of air ventilation and, according to Chao and $\mathrm{Hu}$ (2004), ventilation on demand could save up to $30 \%$ of the energy consumption in a building. Apart from building technology and environmental monitoring, $\mathrm{CO}_{2}$ sensors are applied in food and agriculture industry and as fire detectors. In respect of increasing digitalisation and personal data col- lection, $\mathrm{CO}_{2}$ sensors could also become valuable add-ons in smartphones. This, however, will only be possible on the basis of low cost, reliable, and, especially, small $\mathrm{CO}_{2}$ sensors.

Available $\mathrm{CO}_{2}$ sensors on the market are almost solely of optical nature, based on non-dispersive infrared (NDIR) spectroscopy. Such sensors show high resolution (up to $50 \mathrm{ppm}$ ), high selectivity, high stability, long lifetime, and low maintenance costs as they can be self-calibrating (Wong, 1983; Pandey and Kim, 2007). However, NDIR sensors still have high acquisition costs, which make the omnipresence of these sensors in intelligent buildings unsuitable. They are also limited in the amount their size can be reduced and are thus impractical for mobile phone sensors. Other promising sensor concepts for $\mathrm{CO}_{2}$ based on conductive (Haeusler and Meyer, 1996), electrochemical (Kaneyasu et al., 2000), and mass sensitive (Zhou et al., 1994) detection also exist. All of these sensor types, however, have one or more of the following disadvantages: high operation temperatures above 
$400^{\circ} \mathrm{C}$, lifetime of less than 2 years (Yamazoe and Miura, 1994), and high costs or cross-sensitivities.

The thin film bulk acoustic resonator (FBAR), which is most commonly used for biosensing applications (Wingqvist, 2010), is an acoustic sensor element based on mass sensitivity. Contrary to NDIR and electrochemical sensors, it can be fabricated in very small dimensions and integrated in standardised CMOS (Complementary metal-oxidesemiconductor) micro-fabrication processes. It is a robust device capable of being mass produced, which reduces fabrication costs. Moreover, due to the small size, it is possible to combine a number of sensors to an array to increase functionality. Compared to the well-known Quartz Crystal Microbalances (QCMs), FBARs have much higher resonance frequencies, $f_{\mathrm{r}}$, which increase its sensitivity $S$ according to the equation

$S=-\frac{2 f_{\mathrm{r}}^{2}}{\rho v_{\mathrm{ac}}}$,

where $\rho$ is the density and $v_{\text {ac }}$ is the acoustic-wave velocity of the resonator (Wingqvist, 2010).

FBARs themselves are not sensitive to specific gases and, therefore, have to be equipped with a functional top layer. The functional layer influences selectivity and stability of the final gas sensor and, therefore, has to be chosen carefully. For $\mathrm{CO}_{2}$ the choice of a sensitive, gas selective, and stable material is especially difficult, as $\mathrm{CO}_{2}$ is a rather inert gas and in its size and polarity similar to other atmospheric gases, such as $\mathrm{H}_{2} \mathrm{O}, \mathrm{SO}_{2}$, and $\mathrm{NO}_{x}$. Amino groups $\left(-\mathrm{NH}_{x}\right)$ are well known to interact with $\mathrm{CO}_{2}$ at room temperature, which is why they are extensively used in industrial $\mathrm{CO}_{2}$ capture processes (Bollini et al., 2011). Moreover, amino groups bound in the form of amino-polysiloxanes have the ability to be easily deposited as thin solid layers. All of those properties are the reason why they were considered as functional materials for $\mathrm{CO}_{2}$ detection in the following research.

One major drawback of most solid state sensors, that use $\mathrm{CO}_{2}$ functional materials, are cross-sensitivities with other gases. This problem can be addressed by using multiple FBARs with different functionalisation mixtures or functional layer thicknesses. The method used for distinguishing different gases utilises the detection of changes in the acoustic properties of the functional material additionally to mass changes. In this paper we specifically demonstrate the selection of $\mathrm{CO}_{2}$ from one of the most common cross-sensitivities: humidity. The approach that we introduce is interesting as it does not require any additional sensor set-up, such as preselection membranes (Kaneyasu et al., 2000) or auxiliary electrodes (Sadaoka, 2007), and theoretically can separate any number of gases with the right amount of FBARs. The approach is also useful to determine unknown acoustic parameters of thin layers based on their gas sensitivity. Furthermore, we show that $\mathrm{CO}_{2}$ sensing in the range between 0 and $2000 \mathrm{ppm}$ is possible with a resolution of $50 \mathrm{ppm}$ using FBARs.

\subsection{Working principle of FBAR gas sensors}

The used FBAR set-up is depicted by a cross section in Fig. 1a. It consists of a stack of layers deposited and structured using conventional semiconductor technology. The heart of a FBAR is always a piezoelectric thin film (in this case $\mathrm{ZnO}$ ) sandwiched between two electrodes. When an AC voltage is applied on the electrodes, an acoustic wave originating from the oscillating piezoelectric material is excited. The acoustic wave gets reflected on interfaces that have a large difference in their acoustic impedances. On the top side of the FBAR, the interface to air is an almost perfect reflector. On the bottom side, on the other hand, an acoustic Bragg reflector of alternating materials with high and low acoustic impedances (in this case tungsten, $W=54.7 \mathrm{MPa} \mathrm{s} \mathrm{m}^{-1}$, and silicon dioxide, $\mathrm{SiO}_{2}=8.3 \mathrm{MPa} \mathrm{s} \mathrm{m}^{-1}$ ) is created. Above the top electrode, the functional layer is deposited, which is designed to adsorb specific gases. Originating from the development of the FBAR as a biosensor (Weber et al., 2006), we use $c$ axis-inclined $\mathrm{ZnO}$ as a piezoelectric material, which can generate shear mode waves that dissipate less in viscous media.

Depending on the thickness and acoustic parameters of the FBAR layers, a resonance frequency, $f_{\mathrm{r}}$, can be determined. Resonance only occurs when the half-wavelength $(\lambda / 2(2 n+1)$, with $n$ being the number of over modes) is equal to the resonator thickness. In Fig. $1 b$ the resonance frequency is shown qualitatively in a graph. In the case of gas absorption in the functional layer, the total mass of the resonator increases, which causes a decrease in $f_{\mathrm{r}}$. Assuming that the expansion of the functional layer with gas absorption is negligible, we can define a direct dependence between mass change, $\Delta m$, and density change, $\Delta \rho$ :

$\Delta m=\Delta \rho \times t \times A$,

where $t$ is the thickness of the functional layer and $A$ the area of the resonator. However, gas absorption does not only change the resonator mass but also can have effects on the acoustic velocity, $v_{\mathrm{ac}}$, of a material. The following equation describes the correlation of $v_{\mathrm{ac}}$ to the material parameters stiffness, $c$, and $\rho$ :

$c=v_{\mathrm{ac}}^{2} \times \rho$,

$\Delta c=v_{\mathrm{ac}, 2}^{2} \times \rho_{2}-v_{\mathrm{ac}, 1}^{2} \times \rho_{1}$.

The acoustic velocity, and therefore the stiffness, of a material can increase with gas absorption if, for example, chemical bonds induce a cross-linking in the material. This can be observed in a positive resonance frequency shift as shown in Fig. 1b.

\subsection{Functional material for $\mathrm{CO}_{2}$ adsorption}

As previously mentioned, FBARs need a functional layer that interacts with $\mathrm{CO}_{2}$ in order to change any properties that can 

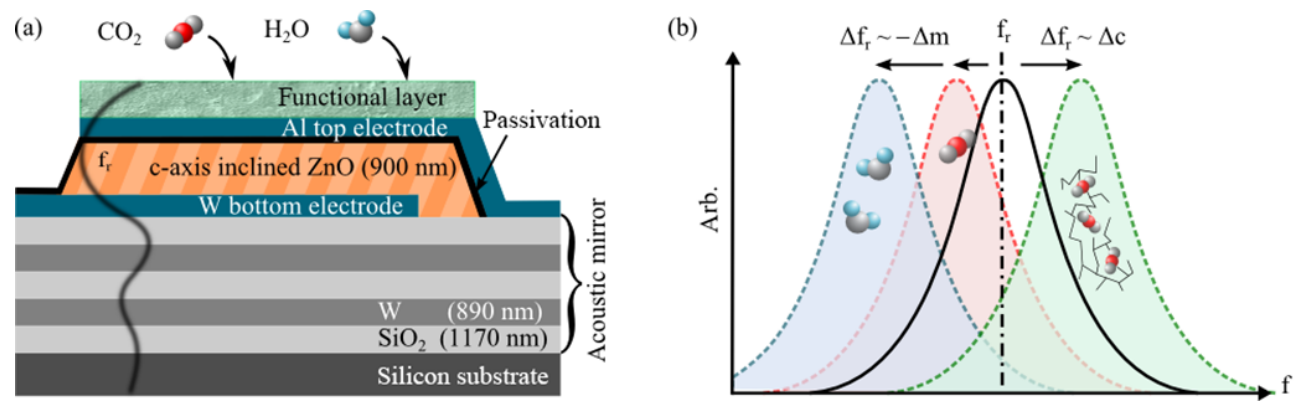

Figure 1. (a) Set-up of the solidly mounted FBAR as used in this work. The functional layer is designed to adsorb $\mathrm{CO}_{2}$ and $\mathrm{H}_{2} \mathrm{O}$ gas. (b) Shift of the resonance frequency $\left(f_{\mathrm{r}}\right)$ to lower frequencies if the sensor mass increases $(\Delta m)$ due to gas absorption, and to higher frequencies if the sensor materials get stiffer $(\Delta c)$.

be measured. For selective mass sensing the requirements towards the functional material are fast gas diffusion, affinity to the desired gas, mechanical and chemical stability, reversibility of adsorption, and good processability. Material groups that are known for $\mathrm{CO}_{2}$ adsorption were summarised by Choi et al., 2009. As stated before, amine-based organic compounds are one of the most promising materials amongst this group. Amines can bind $\mathrm{CO}_{2}$ through acid-base reactions. In anhydrous atmospheres, 2 moles of amine are necessary to bind 1 mole of $\mathrm{CO}_{2}$ in the form of carbamate (see Fig. 2). In hydrous atmospheres, water can act as an additional free base and thus only one amino group is needed to bind $\mathrm{CO}_{2}$ and form bicarbonate species. The kinetics of these reactions in different environments has been studied thoroughly but is not yet fully understood (Choi et al., 2009).

In amino-polysiloxanes, short-chained amino groups (e.g. aminopropyl) are connected to a network of polymeric silicon oxide. The material can easily be applied through wetdeposition techniques (e.g. spin coating, drop coating, printing), is non-toxic, has good gas diffusion properties, and the core siloxane network is chemically inert. Moreover, Yu et al., 2009 found reversible cross-linking in aminopolysiloxanes, which could be advantageous for stiffness measuring FBARs.

\section{Experimental section}

\subsection{FBAR structures}

In Fig. 3, the structure of two FBAR designs are shown. One design has an integrated CMOS readout circuit (active FBARs Fig. 3b) while the other one consists of FBARs without readout that have to be analysed using a network analyser (passive FBARs Fig. 3a).

The passive FBARs (14 in a row) are designed with long contact lines $(750 \mu \mathrm{m})$ to measure the acoustic impedance while the FBARs are surrounded by a gas flow cell. In this design, only one FBAR can be measured at a time but the full impedance characteristics (magnitude and phase) are accessible. We used passive FBARs for most experiments, as

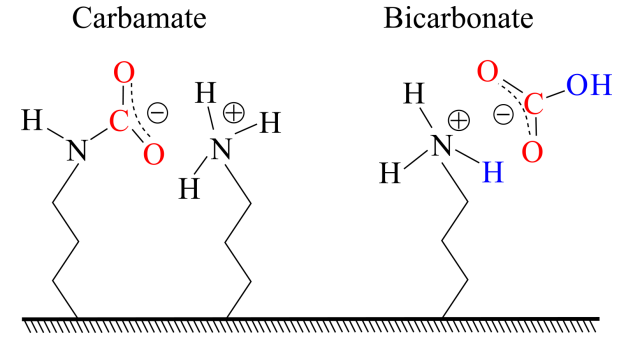

Figure 2. The two most common interactions of $\mathrm{CO}_{2}$ with amines. Carbamate needs two amine groups in the vicinity, whereas bicarbonate utilises water as a free base.

(a)

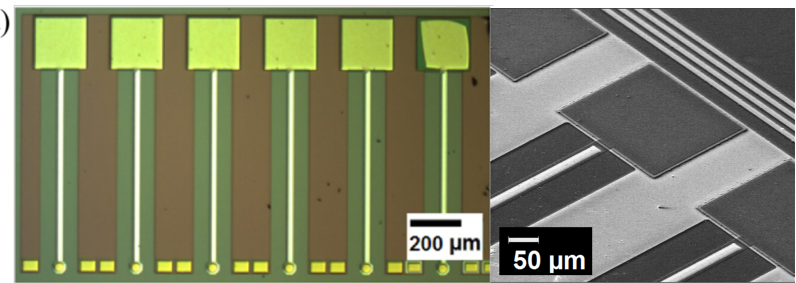

(b)

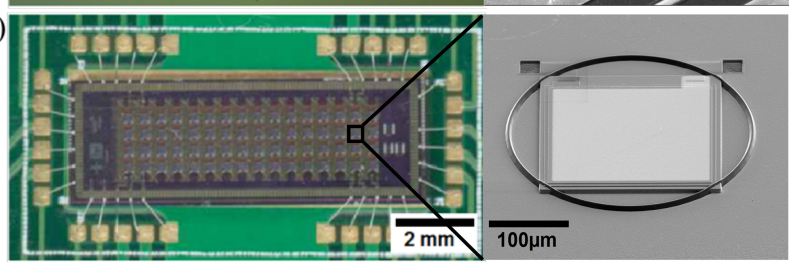

Figure 3. (a) Left: simple passive FBAR structure (top view) with long contact lines enabling the application of a flow cell for gas measurements. Right: SEM image of passive FBARs. (b) Left: CMOS integrated 64-FBAR sensor array. Right: SEM image of a single active FBAR with the confinement ring for micro-drop deposition visible.

they can be produced cheaper and quicker than active FBARs and give detailed information about how materials and gases change the acoustic resonator impedance. Shear resonance frequencies were around $800 \mathrm{MHz}$.

The active FBAR array consists of 64 FBARs $(4 \times 16)$ that can all be read out simultaneously by the CMOS circuit and 
thus changes in $f_{\mathrm{r}}$ can be monitored parallel and evaluated statistically. The active readout also leads to a higher $S / N$ ratio. As a drawback, no detailed impedance characteristic can be obtained. The active FBARs were originally designed with a ring structure around each sensor for individual microdrop deposition, as can be seen in the SEM (scanning electron microscope) image on the right sight of Fig. $3 b$.

\subsection{Functionalisation process}

As $\mathrm{CO}_{2}$ sensitive materials, we chose polysiloxanes derived from three different monomers: 3aminopropyltrimethoxysilane (AMO), propyltrimethoxysilane (PTMS), and 3-aminopropyldiethoxymethylsilane (A2EO) through hydrolysis and subsequent condensation reactions. All monomers were purchased from ABCR GmbH \& Co. KG, Germany, with a purity of $>96 \%$. The hydrophobic PTMS was mixed with the functional aminosilanes AMO and A2EO to reduce humidity cross-sensitivity. The substances AMO and A2EO differ in the dimension of their linkages. AMO can form three-dimensional (3-D) siloxane networks, whereas A2EO forms only 2-D networks and thus results in more viscous polymers. The derived polymer mixtures were dissolved in methanol in different concentrations. For the sensing of humidity, a solution of ethyl cellulose in butanol was prepared by stirring for $1 \mathrm{~h}$ at $80^{\circ} \mathrm{C}$. An overview of all solution compositions and concentrations used in this work is given in Table 1.

For passive FBARs, the solutions were applied in a dynamic spin-coating process at $5000 \mathrm{rpm}$ for $60 \mathrm{~s}$. Due to different concentrations, the resulting layer thickness could be controlled between 0 and $500 \mathrm{~nm}$, as is shown in Fig. 4a for pure AMO for example. The thickness of the functional layers was determined via a KLA-Tencore P-17 profilometer with a maximum deviation of about $\pm 10 \%$ (Fig. 4b). After spin coating, the coated FBARs were annealed for $18 \mathrm{~h}$ at $80^{\circ} \mathrm{C}$ to evaporate the solvent and fully polymerise the siloxanes.

For active FBARs the objective was to deposit single or different polymers of various thicknesses on a single chip. As this is not possible with spin coating, a drop-coating technique was applied. First, the middle of a chip, as depicted in Fig. 3b, was vertically covered with a thin Kapton tape. Then a $0.4 \%$ solution of P2 and P3 was dropped on the left and the right sides, respectively. Although this way of coating is not fully reproducible, it was sufficient for first statistical analyses of locally differing functionalisations. The profile of one row of FBARs after drop deposition is shown in Fig. 5.

\subsection{Gas measurements}

For gas measurements, a synthetic base gas of $20 \% \mathrm{O}_{2}$ and $400 \mathrm{ppm} \mathrm{CO}_{2}$ in a $\mathrm{N}_{2}$ stream with $40 \%$ relative humidity (RH) was chosen, mixed by gas flow controllers (accuracy about $3-4 \%$ ). To analyse the gas response of the sensor, hu-
Table 1. Solutions of polymerised aminosilanes and ethyl cellulose that were prepared for functionalising FBARs.

\begin{tabular}{lrrrrr}
\hline Name & AMO & PTMS & A2EO & Cellulose & $\begin{array}{r}\% w / w \text { in } \\
\text { methanol }\end{array}$ \\
\hline P1 & 65 & 30 & 5 & - & $0.1-14$ \\
P2 & - & 70 & 30 & - & 0.4 \\
P3 & - & 30 & 70 & - & 0.4 \\
C & - & - & - & 100 & $1-3$ \\
\hline
\end{tabular}
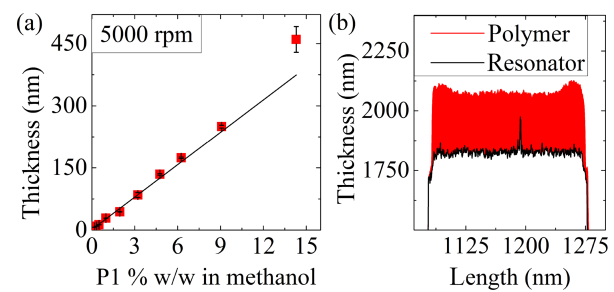

Figure 4. (a) Polymer thickness after spin coating of P1 solution at $5000 \mathrm{rpm}$ for different dilutions. (b) Profile of coated and uncoated FBAR measured with profilometer to determine the thickness of the functional layer.

midity and $\mathrm{CO}_{2}$ concentrations were changed while keeping the flow rate constant at $1 \mathrm{~L} \mathrm{~min}^{-1}$. The gas stream was led through a gas flow cell designed to cover the FBARs. To minimise environmentally caused drifts, the set-up was placed on top of a temperature chuck with a controlled temperature of $25^{\circ} \mathrm{C}$. The resonance frequency and impedance characteristics of passive FBARs were measured using an Agilent 8720ES network analyser from Hewlett Packard, whereas the resonance frequencies of active FBARs were obtained from the integrated CMOS circuit and reader unit, as graphical software LabVIEW was used.

\section{Influences of the FBAR sensitivity}

\subsection{Simulations}

As stated above, FBARs can detect changes in mass as well as changes in the stiffness of materials. From Eqs. (2) and (4), we can deduce that both parameters depend mainly on density, $\rho$, and the acoustic velocity, $v_{\mathrm{ac}}$, of the resonator materials. Both values can be changed by the adsorption of a gas in the functional layer $\left(\Delta v_{\mathrm{ac}}, \Delta \rho\right)$. Using the Mason model for $c$ axis-inclined $\mathrm{ZnO}$, derived in the work of Link (2006), implemented in MATLAB, we calculated the resonance frequency shift normalised to the initial resonance frequency $\left(\left(f_{\mathrm{r}}-f_{\mathrm{r} 0}\right) / f_{\mathrm{r} 0}\right)$ of an FBAR based on certain gas-induced $\Delta v_{\mathrm{ac}}$ and $\Delta \rho$ for changing functional layer thickness. The model uses a simplified FBAR stack of $890 \mathrm{~nm} \mathrm{~W}, 800 \mathrm{~nm} \mathrm{ZnO,} 60 \mathrm{~nm} \mathrm{SiO}_{2}, 200 \mathrm{~nm} \mathrm{Al}$, and 0 $550 \mathrm{~nm}$ polymer (from bottom to top). The tungsten and aluminum layers form the bottom and top electrode, respec- 


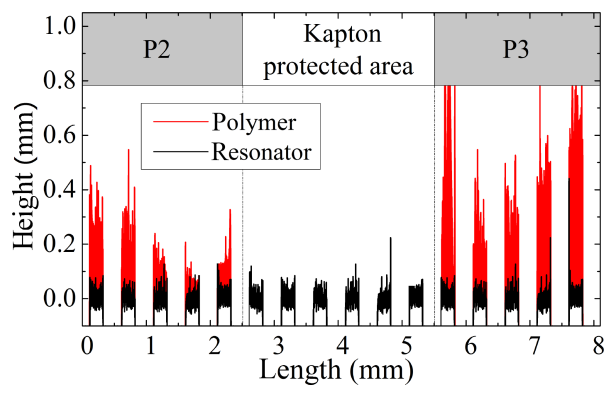

Figure 5. Profile of 16 FBARs in a row on an active sensor chip with the left side functionalised with $\mathrm{P} 2$ and the right side with $\mathrm{P} 3$ after the use of drop deposition. A Kapton tape in the chip middle was used for polymer separation.

tively. For the functional layer, we assumed initial parameters of $\rho=1700 \mathrm{~kg} \mathrm{~m}^{-3}, v_{\mathrm{ac}}=1000 \mathrm{~m} \mathrm{~s}^{-1}$ and a quality factor $Q=50$, which are within typical ranges for viscoelastic polysiloxanes. The coupling constant of $\mathrm{ZnO}$ was set to 0.18 . Values for $\Delta v_{\mathrm{ac}}$ and $\Delta \rho$ were chosen arbitrarily for the purpose of showing trends without consideration of their dependency on each other.

In Fig. 6, three different examples of calculated normalised resonance frequency shifts for this model are depicted, which are dependent on the functional layer thickness. In each example, certain $\Delta \rho$ and $\Delta v_{\text {ac }}$ with gas uptake were assumed. In the first case (blue line), $\rho$ increases by about $1 \%$ due to gas adsorption, while $v_{\text {ac }}$ stays constant. From Eq. (2), we know that density increase is proportional to mass increase, which induces a negative resonance frequency shift. This trend can be observed up to a thickness of about $280 \mathrm{~nm}$. An increasing density at constant $v_{\text {ac }}$, however, must also lead to a stiffness increase, according to Eq. (4). Increasing stiffness, on the other hand, enhances the resonance frequency, which opposes the negative frequency shift from the mass increase. The positive frequency shift from the stiffness increase only becomes visible at a layer thicknesses above $280 \mathrm{~nm}$ as the fraction of the acoustic wave that penetrates the functional material increases. At thin functional layer thicknesses the mass effect dominates.

In a second scenario (red line), only $v_{\mathrm{ac}}$ increases by about $1 \%$, whereas the density of the functional layer stays constant. Without a density increase, there is no contribution to a negative resonance frequency shift and only Eq. (4) has to be considered. In this equation, the stiffness change is dependent on the quadratic term of the acoustic velocity. The red curve demonstrates nicely how the resulting resonance frequency shift is very small for thin functional layers and then increases rapidly until saturation. Saturation occurs due to a decreased coupling between the piezoelectric layer and the rest of the resonator at high functional layer thicknesses.

The third case (yellow line), is the most likely one for real systems, where both parameters change simultaneously. In

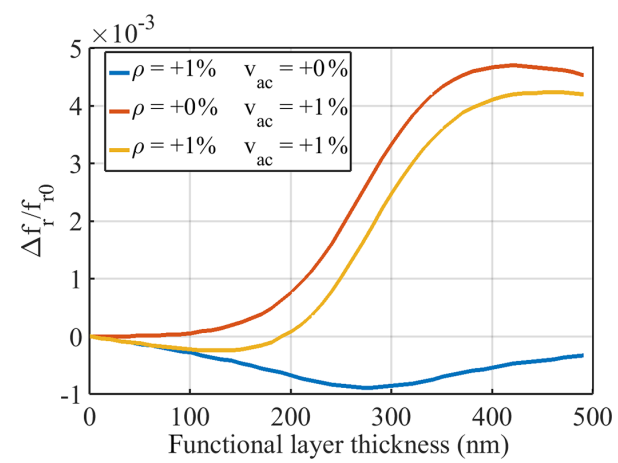

Figure 6. Based on the Mason model, simulated resonance frequency shifts for specific $\Delta \rho$ and $\Delta v_{\text {ac }}$ with gas uptake dependent on the thickness of the functional layer.

this case an inversion of the resonance frequency shift can be observed once the influence of $\Delta v_{\mathrm{ac}}$ exceeds that of $\Delta \rho$. The thickness at which inversion occurs is dependent on the functional material (initial $v_{\mathrm{ac}}, \rho$ ) as well as the interaction with a specific gas $\left(\Delta v_{\mathrm{ac}}, \Delta \rho\right)$. Therefore, the ability of a gas to stiffen a material, in addition to increase its mass, can be used to distinguish between different gases that would otherwise result in the same mass increase. Differences in $\Delta v_{\mathrm{ac}}$ and $\Delta \rho$ for different gases, however, can only be analysed if at least two different thicknesses of a functional layer are available. This fact underlines the advantages of a sensor array consisting of multiple FBARs that can all be coated with a different functional layer thickness.

\subsection{Humidity sensing with ethyl cellulose}

For first principle studies of the above-stated dependency, we deposited various layer thicknesses of ethyl cellulose on FBARs. We chose ethyl cellulose because it is a rather inert material that only shows affinity to humidity, but not $\mathrm{CO}_{2}$, and can be deposited via spin coating in a homogeneous and controlled way. After layer deposition and annealing, we measured the resonance frequency of the FBARs in synthetic air with 40,60 , and $80 \% \mathrm{RH}$ while keeping the temperature constant at $25^{\circ} \mathrm{C}$. Figure 7 exemplarily shows the resonance frequency of two FBARs at different relative humidities. One is coated with $115 \mathrm{~nm}$ and the other one with $235 \mathrm{~nm}$ ethyl cellulose and, as expected, they show a different behaviour due to their different functional layer thickness. The FBAR with the thinner layer has a negative resonance frequency shift with increasing $\mathrm{RH}$, while the thicker layer shows a positive shift. Based on the scale of the graph it can be noticed that the thicker layer also has a larger absolute frequency shift $(\sim 800 \mathrm{kHz}$ for $40-80 \% \mathrm{RH})$ compared to the thin layer ( $\sim 200 \mathrm{kHz}$ for $40-80 \% \mathrm{RH})$ as well as higher noise. The resulting $S / N$ ratio, however, is comparable.

We recorded those resonance frequency shifts as shown in Fig. 7 for about 50 FBARs of different ethyl cellulose thick- 


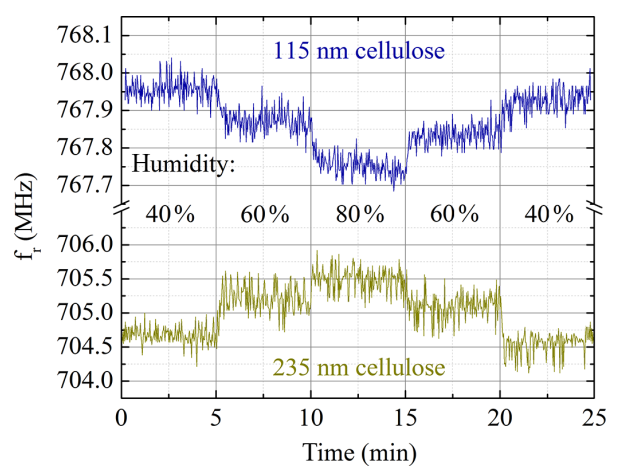

Figure 7. Resonance frequency of two FBARs with different ethyl cellulose thicknesses at 40,60, and $80 \%$ RH.

nesses. The resonance frequency was determined by averaging the last $4 \mathrm{~min}$ of each humidity step. The results of the frequency shift for a humidity change from 40 to $80 \% \mathrm{RH}$ are presented in Fig. 8. This figure also includes the calculated curve from the fitted acoustic parameters of ethyl cellulose at 40 and $80 \%$ humidity. For this fit we used the functional layer thickness and the resonance frequency before and after coating and fitted $\rho$ and $v_{\mathrm{ac}}$ of the functional layer in a way that yields the smallest RSS (residual sum of squares) with the Mason model for all FBARs. The results for $\rho$ and $v_{\mathrm{ac}}$ at both humidity conditions are also presented in Fig. 8 .

The trend of the resonance frequency shift agrees with the simulation case for increasing $\Delta v_{\mathrm{ac}}$ and increasing $\Delta \rho$ from the last chapter. Accordingly, we can deduce that water uptake increases the resonator mass and also the ethyl cellulose stiffness. Looking at the measured data, an increased data scattering can be observed for higher thicknesses. This is caused by several factors. First, there is a statistical error of about $10 \%$ for profilometer measurements. This is partly due to inaccurate levelling and partly due to inhomogeneous coating thicknesses across the FBAR. Second, with increasing layer thickness the acoustic-wave damping increases. This leads to smaller and thus broader resonance peaks and ultimately increases the measurement error of the resonance frequency. However, those two uncertainties might not be sufficient to explain the large deviation of some measurement points from the simulated data. A third possible reason could be inhomogeneities in the acoustic parameters of the deposited material. For example, depending on which data set is used for fitting, the calculated density can get as high as $2100 \mathrm{~kg} \mathrm{~m}^{-3}$ and the acoustic velocity as low as $800 \mathrm{~m} \mathrm{~s}^{-1}$. Considering the many parameters that are involved in the fabricating process (spin-coating velocity, annealing time and temperature, humidity content in air during the processing), it is possible that acoustic parameters slightly differ on each sensor chip. However, despite the deviations, we got a rough estimation about the density and acoustic velocity of a thin layer of ethyl cellulose and could prove that both parameters increase with increasing humidity.

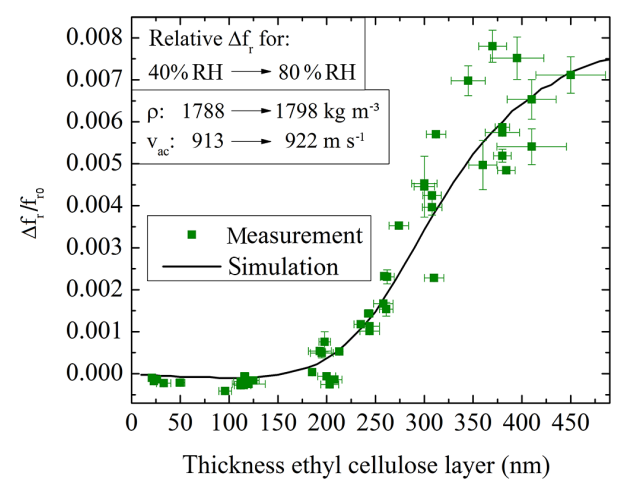

Figure 8. Measured and fitted resonance frequency shift of ethyl cellulose coated FBARs of different thicknesses for a humidity increase from 40 to $80 \% \mathrm{RH}$, always measured after 5 min adjusting time.

\subsection{Humidity and $\mathrm{CO}_{2}$ sensing with amino-polysiloxanes}

Amino-polysiloxanes are polymers sensitive to $\mathrm{CO}_{2}$ and humidity. We deposited different thicknesses of the material on passive FBARs and sensed the response towards changes in $\mathrm{CO}_{2}$ concentration and relative humidity. One example of a $140 \mathrm{~nm}$ thick polymer measured at $25^{\circ} \mathrm{C}$ is depicted in Fig. 9 . The sensitivity towards $\mathrm{CO}_{2}$ was also measured at $70^{\circ} \mathrm{C}$ to investigate how the temperature influences the material-gas interaction. Unfortunately, the humidity cross-sensitivity can not be measured reliably at $70^{\circ} \mathrm{C}$ as the temperature difference between gas and chip changes the resulting relative humidity on the sensor. All measured data are summarised in Fig. 10. A humidity increase from 40 to $60 \%$ (blue data) shows negative sensitivity over the whole range of polymer thickness with slight saturation at the end. This trend is very different compared to cellulose, which had an inversion point at around $100 \mathrm{~nm}$. We conclude from this result that water adsorption has little influence on the stiffness of the polysiloxane, whereas cellulose stiffens with humidity increases.

For $\mathrm{CO}_{2}$, however, an inversion point can be observed at around $220 \mathrm{~nm}$ at room temperature (red data). This may be caused by an increase in stiffness due to carbamate formation, which cross-links the polymer (Yu et al., 2009). A different behaviour was found for the same $\mathrm{CO}_{2}$ increase when measured at $70^{\circ} \mathrm{C}$ (black data). At high temperature, the resonance frequency is lower than at room temperature due to a softening of the polymer and thus decreasing $v_{\mathrm{ac}}$. Moreover, the density might be smaller compared to room temperature because some layer expansion is to be expected and some adsorbed components might evaporate. With low $v_{\mathrm{ac}}$ and $\rho$ the relative increase of both, with the introduction of high- $\mathrm{CO}_{2}$ concentrations, leads to a strong stiffness increase (Eq. 3) and thus to a large resonance frequency shift of up to 2 orders of magnitude compared to room temperature measurements. This increased sensitivity at slightly higher tem- 


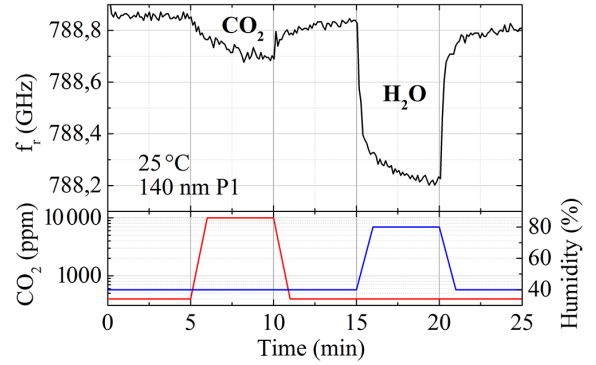

Figure 9. Resonance frequency of an amino-polysiloxanefunctionalised FBAR at different gas concentrations of $\mathrm{CO}_{2}$ and $\mathrm{H}_{2} \mathrm{O}$.

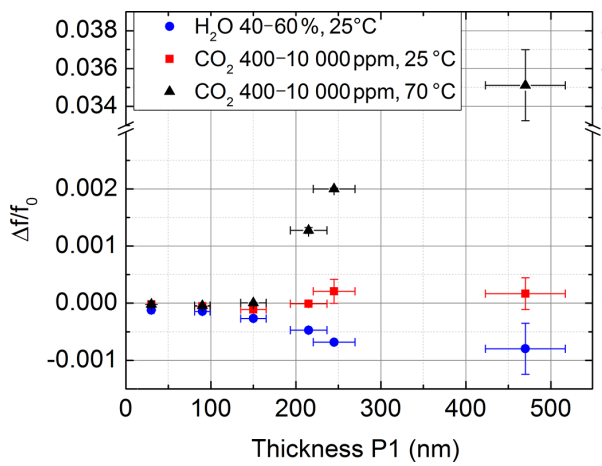

Figure 10. Resonance frequency shifts of amino-polysiloxane mixture $\mathrm{P} 1$ at different thicknesses towards humidity $(40-60 \% \mathrm{RH})$ and towards $\mathrm{CO}_{2}(400-10000 \mathrm{ppm})$ at 25 and $70{ }^{\circ} \mathrm{C}$.

peratures could be useful to increase the total sensitivity of an FBAR-based $\mathrm{CO}_{2}$ sensor. Furthermore, the different changes of the acoustic parameters at different temperatures could be used as another tool to specify different gases. So, instead of two FBARs with different functional layer thicknesses, two FBARs operated at different temperatures but with the same functionalisation could give information about the sensed gas species.

\section{Demonstration of selective $\mathrm{CO}_{2}$ and relative humidity sensing using an FBAR array}

\subsection{Range and resolution}

A sensor array of 64 single FBARs was coated with the aminosiloxane-based materials $\mathrm{P} 2$ and $\mathrm{P} 3$, as shown in Fig. 5 . The thickness of the resulting functional layers varies considerably. P3 was generally found to be deposited with a higher thickness. This might have resulted from slightly different dilutions or material volume during drop deposition. Active FBAR devices have the advantage of measuring multiple FBARs simultaneously and thus obtaining comparable results that can be analysed statistically. Moreover, noise is considerably lower, which makes more sensitive sensing pos-

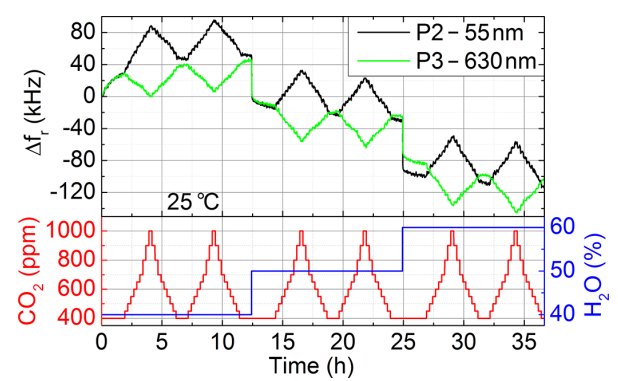

Figure 11. Gas-induced change of the resonance frequency of two FBARs from an active FBAR array. Both FBARs are coated with different polymers of varying thicknesses. Resolutions of $50 \mathrm{ppm}$ were achieved.

sible. In Fig. 11, a detailed gas measurement of two random FBARs from the array is demonstrated in which the $\mathrm{CO}_{2}$ concentration was varied in steps of 50 and later $100 \mathrm{ppm}$ from 400 to $1000 \mathrm{ppm}$ while the relative humidity was changed in $10 \%$ steps from 40 to $60 \%$. Those FBARs achieved a resolution of $50 \mathrm{ppm}$ for $\mathrm{CO}_{2}$. This is an important benchmark, as $75 \mathrm{ppm}$ accuracy is considered a requirement for most indoor air $\mathrm{CO}_{2}$ sensors (Apte, 2006).

Regarding the concentration range, we investigated concentrations between 0 and $10000 \mathrm{ppm} \mathrm{CO}_{2}$, measured at $70^{\circ} \mathrm{C}$ as this yields the highest sensitivities (Fig. 12). In the range between 0 and $2000 \mathrm{ppm}$ the resonance frequency shift increases linearly with the $\mathrm{CO}_{2}$ concentration, whereas above 4000 ppm sensitivity decreases due to saturation of adsorption sites in the volume of the functional layer. As we used passive FBARs for those investigations, the error bars in Fig. 12 are accordingly high. Still, a resolution of up to $200 \mathrm{ppm}$ was achieved for concentrations up to $2000 \mathrm{ppm}$ in this set-up.

\subsection{Selectivity}

The sensitivity of a device can be quenched considerably when other cross-sensitivities affect the sensor signal. In the measurements from Fig. 11, it is visible that both humidity change and change in the $\mathrm{CO}_{2}$ concentration create a resonance shift. Due to the use of two different aminopolysiloxane mixtures (P2 and P3), different resonance shift behaviours are present. The FBAR coated with $630 \mathrm{~nm}$ P3 obviously detects preferably mass changes because gas concentration increases lead to a negative resonance frequency shift (similar to the blue curve in Fig. 6). The material P2, on the other hand, shows an inverse behaviour for increasing $\mathrm{CO}_{2}$ concentration even at a relatively low thickness of $55 \mathrm{~nm}$ (similar to the red curve in Fig. 6), which is clear evidence of an increase of $v_{\mathrm{ac}}$ and thus the stiffness of the functional layer with $\mathrm{CO}_{2}$ uptake. To explain this different behaviour of $\mathrm{P} 2$ and $\mathrm{P} 3$, which are both mixtures of the same materials but with varying ratios, the polymer structure has to be considered (Fig. 13). P2 consists for the largest part 


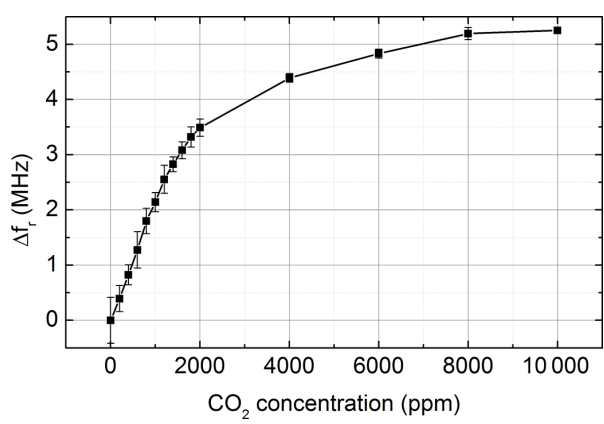

Figure 12. Resonance frequency shift of a P1-functionalised FBAR $(200 \mathrm{~nm})$ at $70^{\circ} \mathrm{C}$ dependent on the $\mathrm{CO}_{2}$ concentration.

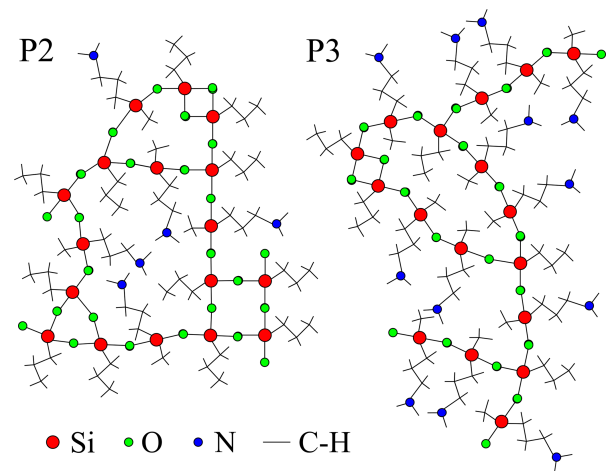

Figure 13. Structure of the amino-polysiloxanes P2 and P3.

of PTMS, which forms stable 3-D networks. Only $30 \%$ of $\mathrm{P} 2$ is made up of $\mathrm{A} 2 \mathrm{EO}$, which connects only in 2-D structures and has functional $\mathrm{NH}_{2}$ groups. If $\mathrm{CO}_{2}$ connects two adjacent $\mathrm{NH}_{2}$ groups forming carbamate, the flexible areas of P2 lose their flexibility, which results in an increased stiffness. The second material, P3, consists of $70 \%$ A2EO and $30 \%$ PTMS. It is thus a more viscous polymer that barely has any stable 3-D network connections. This might make $\mathrm{NH}_{2}$ groups harder to access by $\mathrm{CO}_{2}$. The high viscosity could also prevent the material from stiffening. A further possible explanation could be that the hydrophobicity of PTMS forces the material P2 to adsorb $\mathrm{CO}_{2}$ in the form of carbamate while $\mathrm{P} 3$ can easily form non-cross-linking bicarbonate compounds with adsorbed water molecules.

To demonstrate the selectivity of this $\mathrm{CO}_{2}$ sensor against humidity, we described the relation between the resonance frequency shift and the $\mathrm{CO}_{2}$ and humidity concentrations. For the frequency shift regarding $\mathrm{CO}_{2}$ concentration, we used a logarithmic fit, whereas for humidity, we assumed a linear dependency:

$$
\begin{aligned}
& \Delta f_{\mathrm{P} 2}=a_{\mathrm{P} 2} \times \ln \left(c_{\mathrm{CO}_{2}}\right)+b_{\mathrm{P} 2}+c_{\mathrm{P} 2} \times c_{\mathrm{H}_{2} \mathrm{O}}, \\
& \Delta f_{\mathrm{P} 3}=a_{\mathrm{P} 3} \times \ln \left(c_{\mathrm{CO}_{2}}\right)+b_{\mathrm{P} 3}+c_{\mathrm{P} 3} \times c_{\mathrm{H}_{2} \mathrm{O}} .
\end{aligned}
$$

Solving these equations for the concentration of $\mathrm{CO}_{2}\left(c_{\mathrm{CO}_{2}}\right)$ and humidity $\left(c_{\mathrm{H}_{2} \mathrm{O}}\right)$, the environmental gas concentrations can be recalculated. The results of this calculation for the

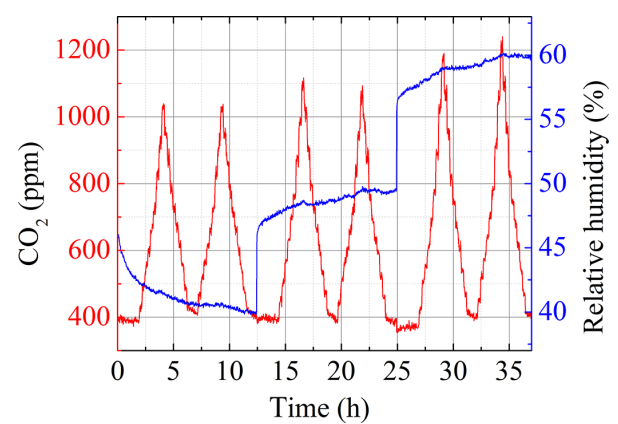

Figure 14. Gas concentrations that were recalculated from the two FBAR responses from Fig. 11 using a two-parameter system.

measurements from Fig. 11 are depicted in Fig. 14. As can be seen, the results correspond quite well to the initial gas concentrations that are shown in the lower graph of Fig. 11. Differences arise due to drifts before the absorption equilibrium, which can be seen especially for the humidity signal, and from the gases influencing each other. For example, the peak of $1000 \mathrm{ppm} \mathrm{CO}_{2}$ at $40 \%$ humidity matches the actual $\mathrm{CO}_{2}$ concentration very well. At $60 \%$ humidity, however, the derived $\mathrm{CO}_{2}$ concentration is $200 \mathrm{ppm}$ higher than the $1000 \mathrm{ppm}$ the sensor was expected to show. This indicates that high humidity benefits from $\mathrm{CO}_{2}$ adsorption in the polymers, possibly due to the formation of bicarbonates, as was also observed by Sayari and Belmabkhout (2010). To eliminate those uncertainties, a calibration of the $\mathrm{CO}_{2}$ sensitivity dependent on different humidities would be necessary. Even without this calibration adaption, we successfully separated $\mathrm{CO}_{2}$ and humidity signals from each other. The maximum deviation of the $\mathrm{CO}_{2}$ signal was $200 \mathrm{ppm}$ due to changing reaction kinetics, and for humidity we reached a maximum deviation of $5 \%$ due to slow reaction times. We demonstrated the separation on this example of two single FBARs with different functional materials. According to the results presented in Fig. 10, a separation of $\mathrm{CO}_{2}$ and humidity could also be achieved through two FBARs of different thicknesses of the same functional material or one FBAR operating at different temperatures, as long as the two gases change their specific resonance frequency shift differently. These considerations show the potential of a 64-FBAR array for reliable multi-gas sensing.

\subsection{Stability}

Regarding sensor stability, two categories can be distinguished: short-term and long-term stability. Based on Fig. 14, short-term stability, which is visible as drifts, can be discussed. Short-term drifts mainly arise due to slow gas diffusion while the functional layer adjusts to a new environment. From Fig. 14, it is apparent that the humidity signal drifts during the whole period of over $12 \mathrm{~h}$. The time constant for humidity changes in amino-polysiloxanes can be fitted using 
an exponential decay function of second order and is strongly dependent on the functional material and the thickness of the functional layer. This shows that for quantitative measurements it is very important to define a set period of time after which all signals are measured. For real environmental measurements, however, decay functions should be known to implement them into the data analysis software.

For $\mathrm{CO}_{2}$, this drift is visible when two sides of the pyramids are compared that should have the same concentration (Fig. 14). But when $\mathrm{CO}_{2}$ decreases from 1000 to $400 \mathrm{ppm}$, the plateaus are at a higher concentration compared to the increasing path. This can also be due to the chemical interaction between $\mathrm{CO}_{2}$ and the amino groups. For decreasing $\mathrm{CO}_{2}$ concentrations, it costs energy to break the chemical bonds in the material, which leads to lower diffusion rates compared to $\mathrm{CO}_{2}$ uptake. Experiments have shown, however, that the signal from $\mathrm{CO}_{2}$ uptake is completely reversible in a time frame of less than 1 day.

A second issue is the long-term stability with irreversible drifts over days and weeks. For the amino-polysiloxanes, we found that baseline drifts are usually the case in the course of the first weeks accompanied by decreasing sensitivity. This can have multiple reasons, from evaporating short-chained polymers over structural deformations, to parasitic gases, such as $\mathrm{SO}_{2}$, which disable the functional amine groups with irreversible bonds (Khatri et al., 2006). Currently, investigations are being done to clarify the ageing process. In general the amino-functionalised FBARs lose about $20 \%$ sensitivity in the first weeks, which stabilises over the next months, and long-term sensitivity above 6 months varies strongly between polymer mixtures and former stressing of the sensor. Ways to adjust for sensitivity changes over long periods would be regular recalibration or reanimation of the sensitive layer through heating, for example.

\section{Conclusions}

In conclusion, we presented the FBAR as a capable room temperature solid state gas sensor for the sensitive and selective detection of $\mathrm{CO}_{2}$. Based on fundamental relations, we simulated the resonance frequency shift's dependency on mass changes and changes in the stiffness of the functional layer. Using ethyl cellulose as humidity sensitive material, we could confirm that these theoretical results are reasonable and can be used for real systems. With amino-polysiloxanes as $\mathrm{CO}_{2}$ and humidity sensitive material, we expanded our system by one gas and found considerable differences in the gas-induced alteration of the acoustic parameters $\left(\rho, v_{\mathrm{ac}}\right)$ of the functional layer. These differences can theoretically be made visible by using two FBARs functionalised with one material of different thicknesses, two FBARs coated with different materials, or one FBAR measured at different temperatures. Using two FBARs coated with different materials, we demonstrated the separation of $\mathrm{CO}_{2}$ and humidity resulting in a resolution of $200 \mathrm{ppm}$ for $\mathrm{CO}_{2}$. Resolutions at constant humidity, however, reached up to $50 \mathrm{ppm} \mathrm{CO}_{2}$ with active FBARs at a background between 400 and $1000 \mathrm{ppm}$.

Apart from using FBARs as gas sensors, they can also be used to determine the acoustic parameters $\left(\rho, v_{\mathrm{ac}}\right)$ of unknown thin layers, which we showed on the example of ethyl cellulose.

For further research, more attention needs to be given to functional material stability and the mathematical reduction of drifts, which were observed for both short and long time periods.

\section{Data availability}

Research data is available upon request to the authors.

Author contributions. Matthias Schreiter carried out experiments from Sect. 4. Romy Hoffmann prepared the manuscript with contributions from all co-authors.

Competing interests. The authors declare that they have no conflict of interest.

Acknowledgements. The presented results could be gathered with the support of the European ESEE ("Environmental Sensors for Energy Efficiency) project (ENIAC JU no. 324284). Chemical support was given by Angelika Tawil.

Edited by: S. Zimmermann

Reviewed by: two anonymous referees

\section{References}

Apte, M. G.: A Review of Demand Control Ventilation, LBNL60170 Report, Lawrence Berkeley National Laboratory, Berkeley, CA, USA, 2006.

Bollini, P., Didas, S. A., and Jones, C. W.: Amine-oxide hybrid materials for acid gas separations, J. Mater. Chem., 21, 15100 15120, doi:10.1039/C1JM12522B, 2011.

Chao, C. Y. H. and Hu, J. S.: Development of a dual-mode demand control ventilation strategy for indoor air quality control and energy saving, Build. Environ., 39, 385-397, doi:10.1016/j.buildenv.2003.11.001, 2004.

Choi, S., Drese, J. H., and Jones, C. W.: Adsorbent materials for carbon dioxide capture from large anthropogenic point sources, ChemSusChem, 2, 796-854, doi:10.1002/cssc.200900036, 2009.

Haeusler, A. and Meyer, J. U.: A novel thick film conductive type $\mathrm{CO}_{2}$ sensor, Sensor. Actuat. B-Chem., 34, 388-395, doi:10.1016/S0925-4005(96)01847-3, 1996.

Kajtár, L., Herczeg, L., Láng, E., Hrustinszky, T., and Bánhidi, L.: Influence of carbon-dioxide pollutant on human well-being and work intensity, Proceedings of Healthy Buildings, 6, 85-90, 2006. 
Kaneyasu, K., Otsuka, K., Setoguchi, Y., Sonoda, S., Nakahara, T., Aso, I., and Nakagaichi, N.: A carbon dioxide gas sensor based on solid electrolyte for air quality control, Sensor. Actuat. BChem., 66, 56-58, doi:10.1016/S0925-4005(99)00411-6, 2000.

Khatri, R. A., Chuang, S. S., Soong, Y., and Gray, M.: Thermal and chemical stability of regenerable solid amine sorbent for $\mathrm{CO}_{2}$ capture, Energ. Fuel., 20, 1514-1520, doi:10.1021/ef050402y, 2006.

Link, M.: Study and realization of shear wave mode solidly mounted film bulk acoustic resonators (FBAR) made of $c$-axis inclined zinc oxide $(\mathrm{ZnO})$ thin films: application as gravimetric sensors in liquid environments, Doctoral Dissertation, Université Henri Poincaré, Nancy I, France, 2006.

Pandey, S. K. and Kim, K. H.: The relative performance of NDIRbased sensors in the near real-time analysis of $\mathrm{CO}_{2}$ in air, Sensors, 7, 1683-1696, doi:10.3390/s7091683, 2007.

Sadaoka, Y.: NASICON based $\mathrm{CO}_{2}$ gas sensor with an auxiliary electrode composed of LiCO3-metal oxide mixtures, Sensor. Actuat. B-Chem., 121, 194-199, doi:10.1016/j.snb.2006.09.019, 2007.

Sayari, A. and Belmabkhout, Y.: Stabilization of amine-containing $\mathrm{CO}_{2}$ adsorbents: dramatic effect of water vapour, J. Am. Chem. Soc., 132, 6312-6314, doi:10.1021/ja1013773, 2010.
Weber, J., Albers, W. M., Tuppurainen, J., Link, M., Gabl, R., Wersing, W., and Schreiter, M.: Shear mode FBARs as highly sensitive liquid biosensors, Sensor. Actuat. A-Phys., 128, 84-88, doi:10.1016/j.sna.2006.01.005, 2006.

Wingqvist, G.: AlN-based sputter-deposited shear mode thin film bulk acoustic resonator (FBAR) for biosensor applications - a review, Surf. Coat. Tech., 205, 1279-1286, doi:10.1016/j.surfcoat.2010.08.109, 2010.

Wong, J. Y.: Self-calibrating carbon dioxide analyzer, Google Patents, US4578762 A, 1983.

Yamazoe, N. and Miura, N.: Environmental gas sensing, Sensor. Actuat. B-Chem., 20, 95-102, doi:10.1016/09254005(93)01183-5, 1994.

Yu, T., Wakuda, K., Blair, D. L., and Weiss, R. G.: Reversibly Cross-Linking Amino-Polysiloxanes by Simple Triatomic Molecules. Facile Methods for Tuning Thermal, Rheological, and Adhesive Properties, J. Phys. Chem. C, 113, 1154611553, doi:10.1021/jp900115g, 2009.

Zhou, R., Vaihinger, S., Geckeler, K. E., and Göpel, W.: Reliable $\mathrm{CO}_{2}$ sensors with silicon-based polymers on quartz microbalance transducers, Sensor. Actuat. B-Chem., 19, 415-420, doi:10.1016/0925-4005(93)01018-Y, 1994. 